\title{
EDITORIAL
}

\section{Clinical culture in radiology: Time to embrace change}

\section{Chander Mohan, SM}

Director, Department of Interventional Radiology, BLK Super Speciality Hospital, New Delhi, India. E-mail: brigcmohan@gmail.com

The ever expanding development of advanced imaging techniques and introduction of newer applications at a fairly rapid pace poses a challenge for the radiologist to stay updated with these advances for optimal utilization as well as be aware of simultaneous clinical developments.

The referring physicians of today need a clinical interface with the imaging specialist and require radiologists who have advanced in depth understanding of the disease processes, provide the best possible diagnoses and advise the appropriate approach to lesions and guide the relevant therapy choice. The specialist neurologist, neurosurgeon, or oncosurgeon is unlikely to rely on a diagnosis made by a radiologist who has had limited exposure in neuroradiology or oncology, respectively, in providing radiology reports describing the findings in a general manner. This has led to a situation, which has somewhat given the opportunity to subspecialist physicians to interpret imaging on their own and also act as primary providers. We are not far away from the time when computer-assisted diagnostic programs will be available for different body parts. If a radiologist reports with observation about signal densities and general findings leaving the "please correlate clinically" phrase to the physician, radiology runs the risk of being pushed to the background.

Therefore the branch of radiology is at cross roads on how to achieve the objectives of the clinically oriented subspecialty from a single radiology department. Clinical experience is must for any radiologist otherwise soon we may find each specialty taking care of its own specialized imaging such as the obstetricians and intensive care unit physicians who have already taken over ultrasound in a number of institutions. This loss of turf wars is due to inadequate clinical culture in radiology.

As a result there has been a tendency in tertiary and teaching hospitals to pursue the development of sub specialty departments in radiology, each formed by a team of radiologists in that subspecialty to maintain pace with the rapidly developing technology with the short update cycles. This has led to better clinical interactions and increased the role of the subspecialist radiologist in ongoing research work, however, it has come at the cost of isolating radiologists from each other creating tunnel-visioned radiologists who have lost their wider body imaging perspective. Moreover, it can pursue the development of sub specialty departments in radiology, each formed and affect round the clock radiology services.

Further the fragmentation of radiologists may encourage the clinicians to set up their own imaging units and propagate the concept of radiologists working in clinical groups. This has many potential disadvantages for the patients. The clinician may focus on the images with a pre-determined clinical diagnosis and may lead to a biased opinion of radiologist. This has led to ongoing debates regarding fragmentation in the field of radiology, challenging the existence of an independent radiology department.

Also the general physician needs help from radiologists to decide which imaging procedure will be most suitable to provide the diagnosis. If general physicians are undertaking primary diagnosis and management of patients, then clinical radiologists should advise the clinicians and undertake the most appropriate examination.

Public awareness of the clinical role of radiology is essential and is reliant on the radiologist's contact with the patients. However, over the past years radiologists have become very busy on their workstations and have become less

This is an open access article distributed under the terms of the Creative Commons Attribution-NonCommercial-ShareAlike 3.0 License, which allows others to remix, tweak, and build upon the work non-commercially, as long as the author is credited and the new creations are licensed under the identical terms.

\begin{tabular}{|l|l|}
\hline \multicolumn{2}{|c|}{ Access this article online } \\
\hline Quick Response Code: & Website: \\
& www.ijri.org \\
\hline
\end{tabular}

Cite this article as: Mohan C. Clinical culture in radiology: Time to embrace change. Indian J Radiol Imaging 2016;26:297-8. 
visible for patients. In fact, patients typically do not choose their radiologist; the referring physician usually makes that choice. Most of the time patients and their diagnostic radiologist never meet and many patients believe that the clinician who actually requested the examination and received the report from the radiologist is actually the physician who has interpreted the study. For many patients, radiologists are considered only providers of a diagnostic test and not as physicians who play a vital role in the decisions that affect them.

The solution to these problems may be achieved by having radiology departments with radiologists who have developed additional expertise in two or three clinical disciplines while providing a general radiology service and complement each other within the department or practice acting as "hybrid-imagers" working in both general and subspecialty imaging.

Another important thing that the radiologists of today can benefit from is by standardizing the reporting formats used in different organ systems. This can help in efficient communication and second opinion between the fellow radiologists as well as the clinicians. All the radiology subspecialties should also come up with standardized reporting formats for different organ systems developed in consultation with the specialist physicians who are the consumers of all the information we provide. It is high time that we eliminate the technical jargon and standardize the reporting format used per organ system basis. Effective and precise communication with clinical colleagues and patients can be a big step in catapulting the radiologist into the patient care team.

In areas where there are ongoing "turf wars," which are increasing by each passing day, subspecialty qualifications may be a requirement. Radiologists in teaching hospitals should sub specialize to provide a tertiary referral service and be at helm of ongoing imaging research. Radiologists should therefore have areas of subspecialty even though functioning as general radiologist most of the time. The radiologists should work together to ensure that patients admitted for interventional procedures should be admitted under interventional radiologist and generate revenue for radiology department.

The importance of clinical training needs to be emphasized with appropriate clinical subspecialty training in the radiology curriculum. Subspecialty trainees should participate in clinical rounds, clinico-radiological \& tumor board meetings. It is time that we incorporate subspecialty rotations in the final year of the postgraduate radiology curriculum where the trainees get to work in their chosen subspecialty where they intend to work in the long run.

Another fundamental requirement to sensitize the exposure of medical students to role of radiology in patient care taught by trained radiologists. Presently, only few radiologists involved in undergraduate training in India. There are no postings in the pre-clinical and clinical years for radiology. The only exposure that a medical student gets is a few weeks during the internship training, often as an elective posting. As a result the field of radiology appears as less attractive to the young medical student.

Perhaps it is the right opportunity for the radiologists to go beyond the dictated and understand the diseases that they are requested to investigate. Therefore, if radiologists want to walk toe to toe with clinician, they need to subspecialize, the extent of which will be determined based on their working circumstances. However, all this must be from the confines of an independent radiology department.

Radiologists can become the protagonists of this change if they position themselves at the center of the clinical decision-making process, acting as imaging consultants, developing clinico-radiological conferences, tumor boards, and conducting imaging rounds. A deep modification of their mindset is needed at all levels, shifting the emphasis from service provision, operations, and efficiency, to a new role where the radiologist will surely shoulder higher clinical responsibilities. 